\title{
ENERGY LOSS MEASUREMENT IN A MWPC
}

\author{
H. FREHSE, F. LAPIQUE*, M. PANTER ${ }^{\dagger}$ and F. PIUZ
}

CERN, Geneva, Switzerland

\begin{abstract}
Experimental results on the influence of angle and cell geometry on pulse height measurements in a multiwire proportional chamber (MWPC) are presented. Two track orientations are mainly considered: (a) tracks parallel to the wire plane and (b) tracks traversing the gap with one wire touched. In case (a), the positive induced pulse effect is analyzed: pulse heights have to be corrected by factors of 1.3-1.7 and wire inefficiency can be found up to $20 \%$, depending on the cell geometry. The saturation effect starts earlier in case (a), but the influence of the angle between track and wire when approaching $90^{\circ}$ is moderate. On the contrary, this influence is very effective in case (b). A detailed study of this phenomenon is then presented with a description of possible mechanisms involved.
\end{abstract}

\section{Introduction}

The energy lost by a particle in a MWPC gap is related to the measured pulse height by a wellknown multiplication factor, the gas amplification.

In detection systems only devoted to localization of a particle trajectory, a well controlled proportionality between these two quantities is not essential, but is required for the devices designed to measure the energy loss.

Therefore, in the following, we will make a distinction between the energy loss spectrum (ELS) and the measured pulse height spectrum (PHS). It is worthwhile, then, to look at the mechanisms of gas multiplication in order to know how two essential properties of the ELS - proportionality and isotropy - might be affected.

We have focused this study mainly on the influence of the track orientation (fig. 1), since nowadays, detection systems must handle long, mostly curved particle trajectories in large solid angles.

Some new geometries, under development, such as the jet chamber'), the time projection chamber $^{2}$ ), imply track orientation mostly parallel to the sense wire plane which deserves to be compared

* University of Grenoble, Grenoble, France.

$\dagger$ Institut für exp. Kernphysik, Universität (TH), Karlsruhe, Germany.

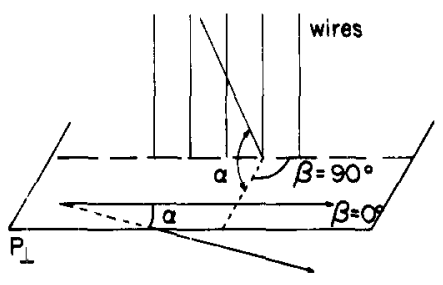

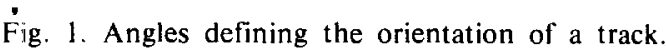

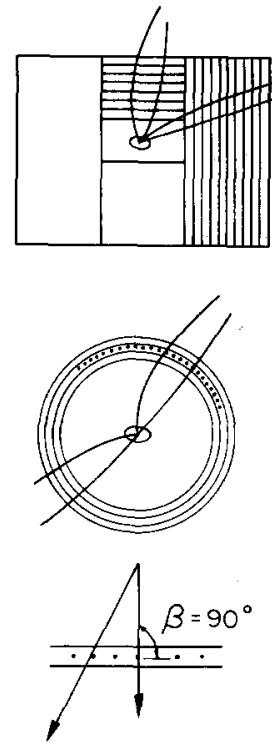

a)
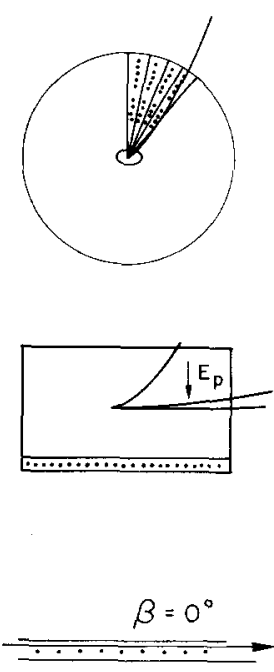

b)
Fig. 2. Some geometries of detection systems. In case (a) trajectories are mostly crossing the sense wire planes $\left(\beta=90^{\circ} \ldots 80^{\circ} \ldots\right)$. In case (b) they are essentially parallel $\left(\beta=0^{\circ}\right)$ up: sketch of a jet chamber geometry.

to the more classical orientation where particles cross the gaps (fig. 2).

\section{Pulse generation}

\subsection{Physical phenomena and parameters}

Since we have to deal essentially with pulse height, let us first recall $^{3}$ ) the sequence of phenomena involved in the pulse generation (fig. 3). They are:

a) Production of primary pairs in a wire cell.

b) Secondary multiplication, generating charges (+ and -) $Q_{0}$ per pair. 


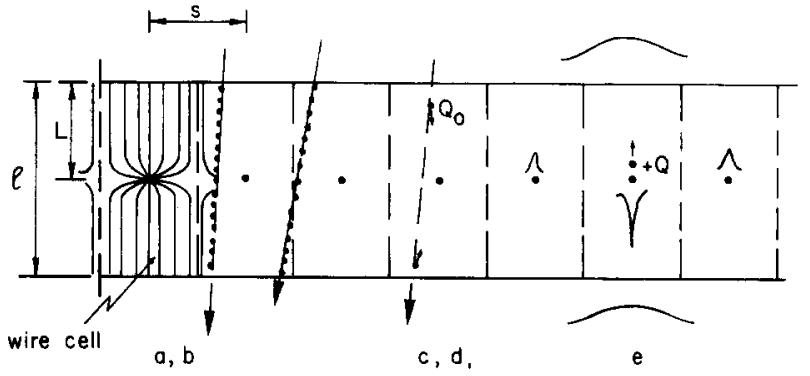

Fig. 3. Definition of cell geometry and pulse generations: L, half gap; 1 , gas thickness; $s$, wire spacing; $\varnothing$, wire diameter; $V$, cathode voltage; (a): a wire cell is the region of the gap which includes all the field lines ending at this wire.

c) Collection of $-Q_{0}$, determined by the electron drift velocity $v_{-}$.

d) Single avalanche process, determined by the gas composition and $E_{0}$ field at the wire surface; generates charges ( + and -$) Q$.

e) Motion of $+Q$, determined by the ion drift velocity; induces signals of opposite polarities on the wire and the neighbouring conductors.

For a given cell geometry and gas mixture, three external parameters have to be considered:

- the applied voltage, $V$,

- the track orientation ( $\alpha, \beta$ of fig. 2$)$,

- the time response of the input circuit and its associated electronics.

These parameters act on the following features:

a) $V$ determines the amount of charges $Q$.

b) The number of wire cells traversed in a gap is fixed by the angle $\beta$.

c) The length of the wire on which the avalanches are collected depends on the angle $\alpha$ at a given $\beta$.

d) The electronic time response influences the pulse shape.

\subsection{Proportional Regime and POSSIBle disturbances}

Measurements are done at the so-called proportional regime when pulse heights $(\mathrm{PH})$ satisfy the equation

$\mathrm{PH}=M \sum Q_{0}$,

where

$\Sigma Q_{0}$ is the total primary charge deposited in the wire cell,

$M$ is the gas amplification defined at the level of a single avalanche process, following ref. 3 ,

$Q=Q_{0} \exp \left[f\left(E_{0}\right)\right]=M Q_{0}$,
$f\left(E_{0}\right)$ accounts for the gas mixture and the cell geometry.

This process occurs in a well defined space and time range. It develops in the vicinity of the wire $(20-100 \mu \mathrm{m})$ and lasts approximately $1-2 \mathrm{~ns}^{*}$.

On the other hand, the summation $\Sigma Q_{0}$ is done over time and space ranges, which are essentially variable and can be large with respect to those involved in a single avalanche process. Therefore, one will find a set of values for the external parameters where eqs. (1) and/or (2) are not fulfilled. For example:

a) Eq. (1), in case of superposition at a same wire of negative and positive induced pulses, that depends on the sharing of $\Sigma Q_{0}$ between several wire cells. This is a pure geometrical effect independent of gas amplification.

b) Eq. (2), in case of an effect of high voltage combined ${ }^{4}$ ) or not with orientation, since the physical phenomena may overlap differently in space and time according to the primary deposition geometry. That will be referred to as the saturation effect.

It is clear that the relative value of the electronic time response, with respect to the durations involved in the physical phenomena, will influence the pulse shape not only on negative signals, but also on the superposition of positive induced signals ${ }^{5}$ ). For example, the relation (1) is valid only for charge measurements, when an expression written for voltage measurements would include some electric parameters.

\section{Remarks on the measurements}

The results were obtained with a beam of $\pi^{-}$, at $1.4 \mathrm{GeV} / \mathrm{c}$ momentum. An external set of MWPC allowed an angular definition of their trajectory with an accuracy of $\pm 0.2^{\circ}$.

The charge sensitive electronics ${ }^{6}$ ) holds the signal levels over a long time compared to the physical duration with a linear dynamical range of $+3 \mathrm{pC}$ to $-16 \mathrm{pC}$. The spectra, measured at different cell geometries and various orientations, were recorded on one wire at a fixed place in the chamber. The gap width can be varied.

The chamber construction provides an overall homogeneity in amplification of $\pm 5 \%$, electronics included. The gas mixture is composed of argon/isobutan/methylal $55 / 38 / 7 \%$ by volume. The

\footnotetext{
* The electrical field near the wire decreases like $E_{0} r_{0} / r$. where $r_{0}$ is the wire radius and $\nu_{-}=20 \mathrm{~ns} / \mathrm{mm}^{3}$ ).
} 
TABLE 1

\begin{tabular}{cccc}
\hline$\beta$ (deg.) & $\alpha$ (deg.) & Overlap in time & Overlap in space \\
\hline 90 & 15 & no & no \\
90 & 0 & no & yes \\
$0^{\text {a }}$ & 15 & yes & no \\
$0^{\text {a }}$ & 0 & yes & yes \\
$\gamma$ & $5.9 \mathrm{keV}$ & yes & yes \\
\hline
\end{tabular}

a Must be corrected for the positive induced pulses effect (sect. 5.3)

means of the pulse height spectra $\langle\mathrm{PH}\rangle$ have been plotted; all results are corrected to $23{ }^{\circ} \mathrm{C}, 730$ torr.

\section{Saturation effect}

In order to disentangle the respective contributions of high voltage and summation process $\Sigma \dot{Q}_{0}$ in the saturation effect, we will consider the influence of the orientation according to the criteria:

- overlap of avalanches in time,

- overlap of avalanches in space.

This leads to the four-geometrical situation, to which we add $5.9 \mathrm{keV}$ photons, listed in table 1 .

\subsection{Pulse shape vs orientation (fig. 4)}

\subsubsection{Overlap in time: $\beta=0^{\circ}$}

In case the distance from the track to the wire is large compared to the wire spacing $\mathbf{s}$, the collection times of the primaries are nearly equal (fig. 4a). All the avalanches develop during a very short time, generating a fast pulse. Fig. 5a shows

a)

$\beta=0^{\circ}$

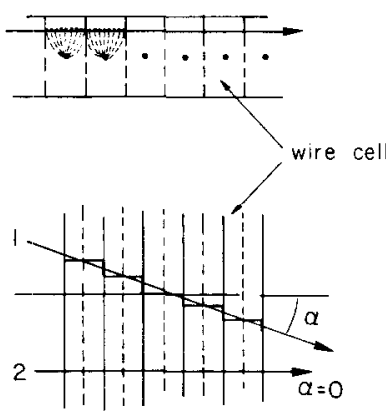

Fig. 4. Schemes of primary charge collection: $(a-1) \beta=0^{\circ}, \alpha$; time overlap; no space overlap. $(\mathrm{a}-2) \beta=0^{\circ}, \alpha=0$; time overlap; space overlap. $(\mathrm{b}-1) \beta=90^{\circ}, \alpha$; no time overlap; no space overlap. $(b-2) \beta=90^{\circ}, \alpha=0$; no time overlap; space overlap. The regions where the avalanches spread along the wire are in a reinforced line. such a shape measured on $470 \Omega$. The time decay is related ${ }^{5}$ ) to the ion drift velocity and the input circuit RC. This pulse shape is also observed with a $5.9 \mathrm{keV}$ photon where all the primary charge is created at a well localized spot in the cell $(100-200 \mu \mathrm{m})$.

\subsubsection{No overlap in time: $\beta=90^{\circ}$}

A large range of collection times is now necessary in order that each primary reaches the wire (fig. 4b). The pulse is generated by a succession of avalanches, delayed in time, up to the maximum collection time $L / v_{-}$. A long pulse is observed, as shown in fig. 5b. The largest local fluctuations in primary energy losses are clearly separated. Such a structure can be observed ${ }^{7}$ ) on collection times up to $4 \mu \mathrm{s}-L=20 \mathrm{~cm}$.

\subsubsection{Overlap in space}

In the two preceding cases, the whole avalanche process may be spread along the wire according to the $\alpha$ value. When $\alpha$ equals $15^{\circ}$, the distance between each avalanche is, on the average, $\approx 80 \mu \mathrm{m}$
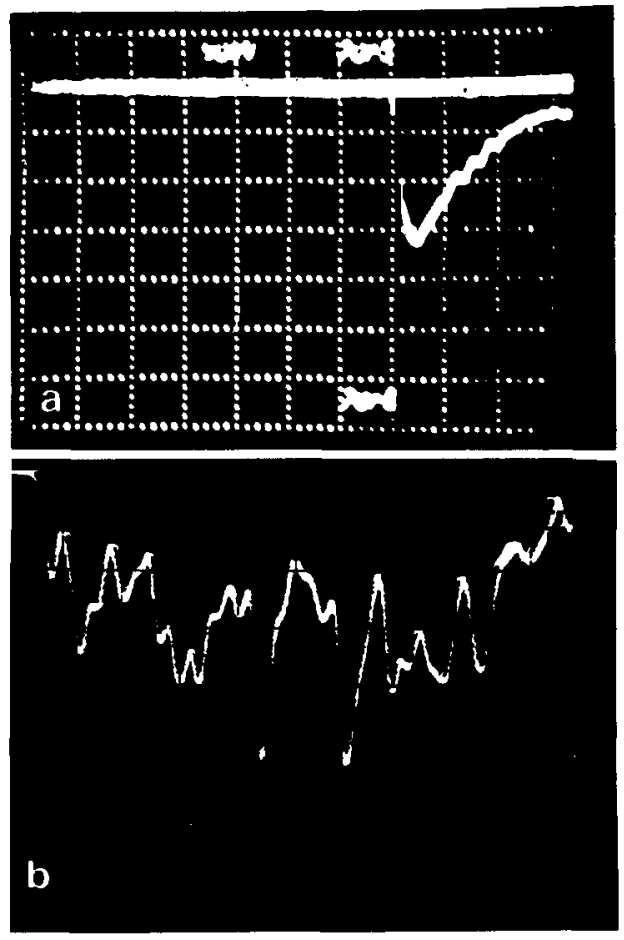

\section{$\longmapsto 20 \mathrm{~ns} /$ division}

Fig. 5. Pulses on $470 \Omega, 20 \mathrm{~ns} / \mathrm{div}$ : (a) $\beta=0^{\circ}, \alpha=15^{\circ}$; amplification $\sim 1 \times 10^{5} \tau_{\mathrm{RC}}$ (circuit) $\approx 2.5 \mathrm{~ns}$; cell: $L=12 \mathrm{~mm}$, $s=4 \mathrm{~mm}$. (b) $\beta=90^{\circ}, \alpha \sim 10^{\circ}$; amplification $\sim 1 \times 10^{5}$; cell: $L=8 \mathrm{~mm} ; v_{-}=20 \mathrm{~ns} / \mathrm{mm}$. 
TABLE 2

Main features of the different pulse generations.

\begin{tabular}{|c|c|c|c|c|c|c|c|c|c|c|}
\hline $\begin{array}{c}\text { Curve } \\
\text { (fig. 6) }\end{array}$ & $\begin{array}{l}\text { Par- } \\
\text { ticle }\end{array}$ & $\begin{array}{c}\beta \\
\text { (deg.) }\end{array}$ & $\begin{array}{c}\alpha \\
\text { (deg.) }\end{array}$ & $\begin{array}{l}\text { Track } \\
\text { length } \\
(\mathrm{mm})\end{array}$ & $\begin{array}{c}\text { Mean } \\
\text { energy } \\
\text { loss } \\
(\mathrm{eV})\end{array}$ & $\begin{array}{c}\text { Number } \\
\text { of } \\
\text { primaries }\end{array}$ & $\begin{array}{c}\text { Mean } Q_{0} \text { per } \\
\text { primary } \\
\text { (number of } \mathrm{e}^{-} \text {) }\end{array}$ & $\begin{array}{l}\text { Number of } \\
\text { avalanche }^{\mathrm{a}}\end{array}$ & $\begin{array}{c}\text { Mean } Q_{0} \text { per } \\
\text { avalanche } \\
\text { (number of } \mathrm{e}^{-} \text {) }\end{array}$ & $\begin{array}{c}\text { Spread } \\
\text { along } \\
\text { the wire } \\
(\mathrm{mm})\end{array}$ \\
\hline 1 & $\gamma$ & - & - & - & 5900 & 1 & 220 & 1 & 220 & $\approx 0.2$ \\
\hline 2 & $\pi^{-}$ & 90 & 15 & 10 & $3000^{b}$ & $35(\mathrm{~m})^{\mathrm{c}}$ & $3^{c}$ & $35(\mathrm{~m})$ & 3 & 2.7 \\
\hline 3 & $\pi^{-}$ & 90 & 0 & 10 & 3000 & $35(\mathrm{~m})$ & 3 & $35(\mathrm{~m})$ & 3 & $\approx 0.2$ \\
\hline 4 & $\pi^{-}$ & 0 & 15 & 4 & 1200 & $14(\mathrm{~m})$ & 3 & 1 & 42 & 1.1 \\
\hline 5 & $\pi^{-}$ & 0 & 0 & 4 & 1200 & $14(\mathrm{~m})$ & 3 & 1 & 42 & $\approx 0.2$ \\
\hline
\end{tabular}

a Not overlapping in time.

b See sect. 4.5 .

c Comes from the Monte Carlo simulation (sect. 4.5).

$\mathrm{m}$ Indicates a mean value.

(table 2). They all overlap in space when $\alpha$ equals $0^{\circ}$. Table 2 summarizes the features of these different pulse generations.

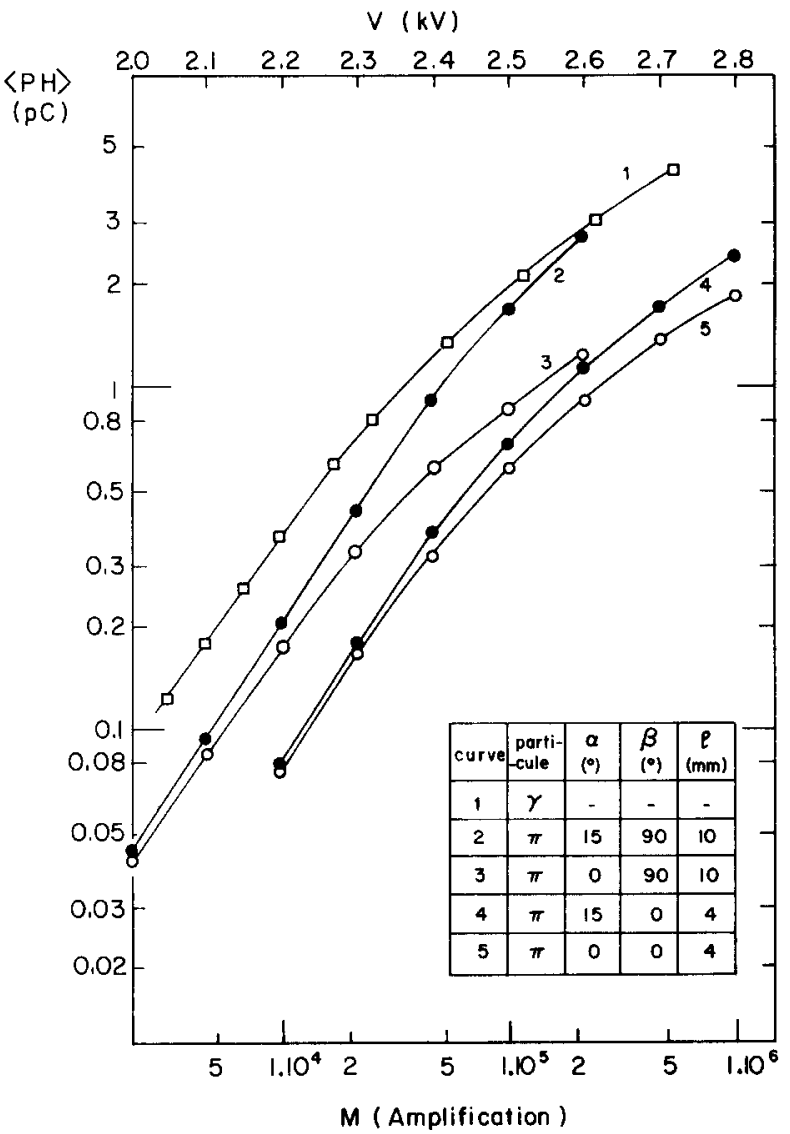

Fig. 6. Pulse height vs high voltage illustrating the different saturation effects. The measurements at $\beta=0$ are corrected from the positive induced pulse effect (sect. 5.2). Cell: $L=5 \mathrm{~mm}, s=4 \mathrm{~mm}$.

\subsection{Pulse height vs orientation}

In fig. 6, we can compare the results obtained for the five situations precedingly described. The following observations can be made:

a) On all curves, an exponential dependence of $\mathrm{PH}$ vs $V$ is observed at low charge, but over different ranges. The slopes are approximately identical. At increasing pulse height, the curves start bending: this new regime is usually called the saturation effect.

b) No space overlap: $\alpha=15^{\circ}$. The longest exponential range in $\mathrm{PH}$ is observed for curve 2, $\left(\beta=90^{\circ}\right)$ up to $\approx 1-2 \mathrm{pC}$ (sect. 4.5 , fig. 12). Saturation occurs earlier $(\approx 0.4 \mathrm{pC})$ for curve $4\left(\beta=0^{\circ}\right)$.

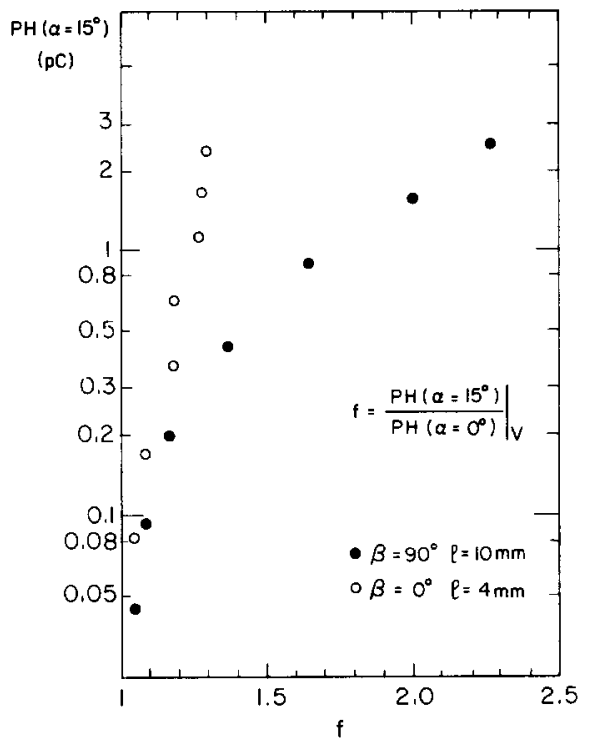

Fig. 7. Saturation factor in both cases $\beta=0, \beta=90^{\circ}$, when $\alpha$ goes from $15^{\circ}$ to $0^{\circ}$. 
c) Effect of space overlap: At $\beta=90^{\circ}$, one sees an important saturation effect by going from curve 2 to curve 3. At $\beta=0^{\circ}$, comparison between curves 4 and 5 does not show such a drastic effect.

In order to illustrate this point (c), we have plotted in fig. 7 the variation of a "saturation factor"

$\left.\frac{\operatorname{PH}\left(\alpha=15^{\circ}\right)}{\operatorname{PH}\left(\alpha=0^{\circ}\right)}\right|_{V}$ versus $\mathrm{PH}\left(\alpha=15^{\circ}\right)$,

in both cases $\beta=0^{\circ}$ and $\beta=90^{\circ}$.
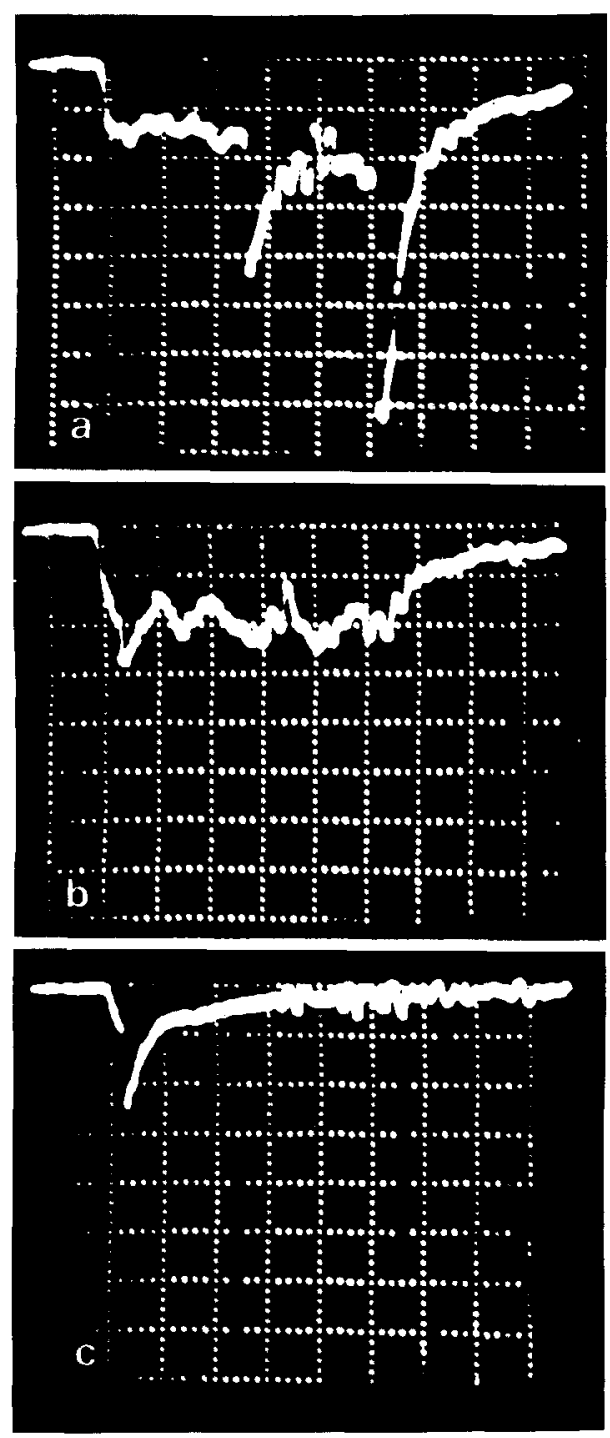

\section{$\longmapsto 50$ 50/division}

Fig. 8. Pulses on $700 \Omega, 50 \mathrm{~ns} /$ div; cell : $L=15 \mathrm{mmn} s=8 \mathrm{~mm}$ : (a) and (b) $\beta=90^{\circ}, \quad \alpha=15^{\circ}$, gas amplification: $3 \times 10^{5}$; $v_{-}=20 \mathrm{~ns} / \mathrm{mm}$. (c) $\beta=90^{\circ}, \alpha=0^{\circ}$; all other conditions unchanged.
This factor increases most rapidly with the measured charge, when space overlap occurs without time overlap.

\subsection{INTERPRETATION OF THE RESULTS}

A tentative explanation might be the following:

\subsection{1. $\beta=90^{\circ}$ and $\alpha=0^{\circ}$}

The avalanches are produced successively each $7 \mathrm{~ns}$, on the average, during the maximum collection time, which is, say, 100-300 ns. Each positive charge $Q$ moves slowly, compared to the primaries; its initial velocity ${ }^{8}$ ) is of the order of few hundred $\mathrm{ns} / \mathrm{mm}$ and decreases with the square root of the field. One can calculate that the positive charge created by the first avalanche has moved from the wire by only $\approx 70 \mu \mathrm{m}$ after $100 \mathrm{~ns}$. Therefore, as $\alpha$ equals $0^{\circ}$, the collection of nearly all the primaries and the complete development of their avalanches occur in a region of even higher positive charge density, that is, where the field is reduced.

\subsubsection{Other orientations}

On the contrary, in the three other cases the situation, where primaries are collected in the middle of a positive cloud, is never realised: one has either a simultaneous collection $\left(\beta=0^{\circ}, \alpha=0^{\circ}\right.$ and $15^{\circ}$ ) or delayed in time, but spread along the wire $\left(\beta=90^{\circ}, \alpha=15^{\circ}\right)$. Therefore, the modification brought by the positive charges, influences only the end of its own avalanche development. The importance of this effect may depend on the total charge present at the same time (curves 2 and 4) or on the charge density (curves 4 and 5).

Fig. 8 illustrates this interpretation. At the experimental conditions of figs. $8 \mathrm{a}, \mathrm{b}$, the maximum collection time is $300 \mathrm{~ns}$ as expected for a gap width of $15 \mathrm{~mm}$. Although the saturated regime is reached, no deterioration of the pulse amplitude is observed during the collection time. In fig. 8c, $\alpha$ equals $0^{\circ}$, all other conditions are unchanged: amplification occurs now in the first 20-50 ns and drops significantly for the remaining $250 \mathrm{~ns}$.

\subsection{Saturation at $\beta=90^{\circ}$ : Dependence on $\alpha^{9}$ )}

This case, where saturation is the most pronounced, was studied in more detail in the cell geometry

$L=5 \mathrm{~mm}, \quad s=4 \mathrm{~mm}, \quad \varnothing=20 \mu \mathrm{m}$. 


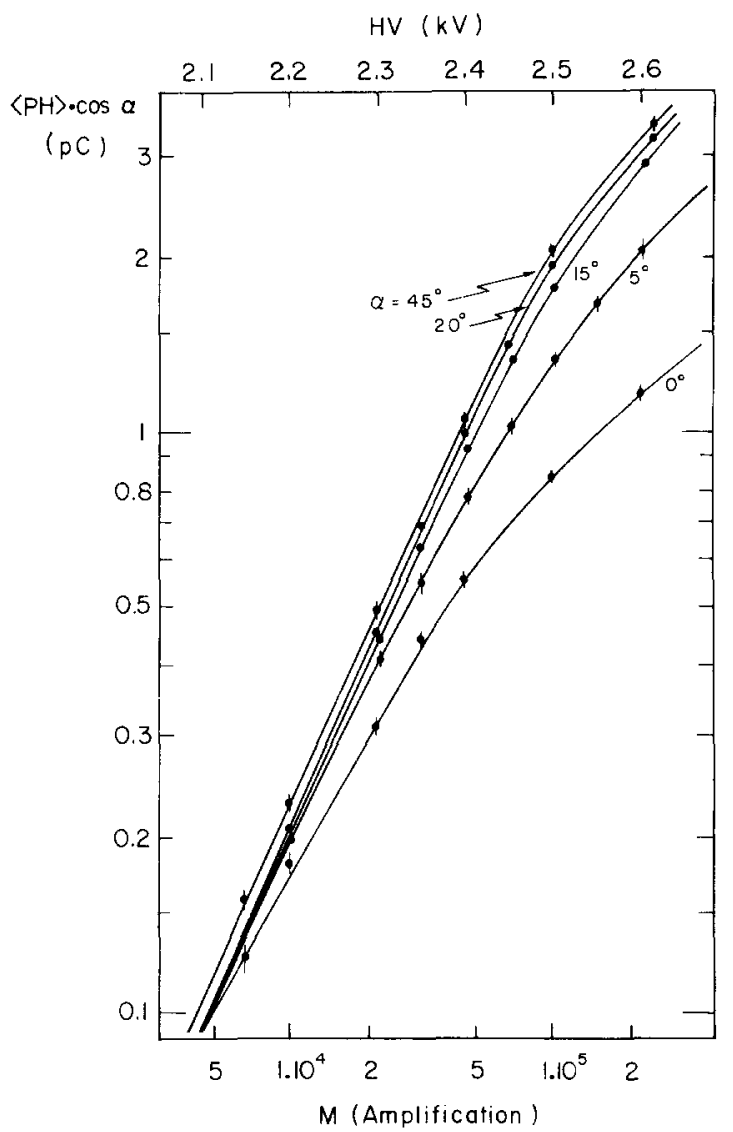

Fig. 9. Pulse height vs high voltage at $\beta=90^{\circ}$ and different $\alpha^{\prime}$; normalized at $l=10 \mathrm{~mm}$; cell: $L=5 \mathrm{~mm}, s=4 \mathrm{~mm}$.

Results are shown in fig. 9. The saturation starts to be effective from $\alpha \approx 20^{\circ}$ and reaches its maximum intensity at $\alpha=0^{\circ}$ (fig. 10).

A $15-20 \%$ effect is still observed at an amplification of $5 \times 10^{3}$ and $10 \mathrm{~mm}$ track length. Nevertheless, the amplification cannot be set at a too low level because the detection of energy losses, as small as possible, is equally wanted in a cell ${ }^{10}$ ): they originate from pieces of inclined tracks or/and from the low energy part of the ELS, representing at minimum one tenth of the mean energy lost through the full gap. This fraction, compared to the equivalent noise level, will mostly determine the amplification. Fig. 11 shows that the energy resolution may depend on the amplification.

\subsection{Proportional regime}

The measurements were performed at $\beta=90^{\circ}$, $\alpha=15^{\circ}$, which fulfills the requirements to get data in this regime (sect. 2.2):

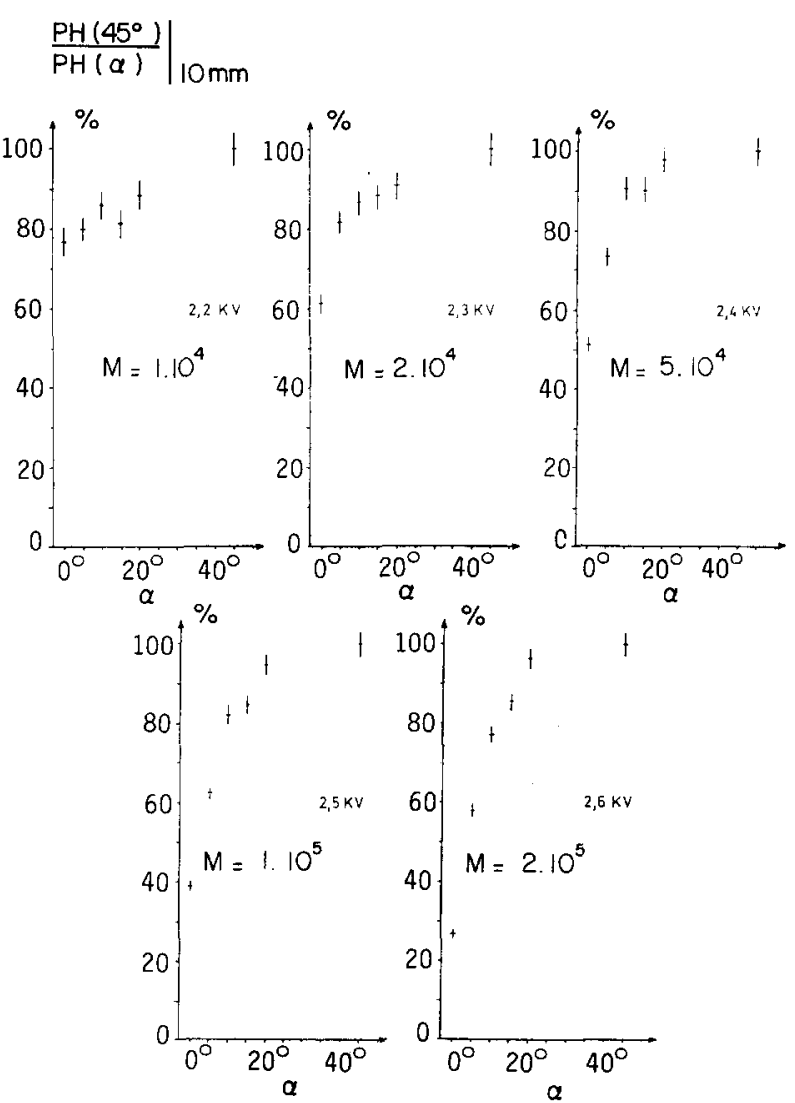

Fig. 10. Saturation effect vs $\alpha$ at different gas amplification at $\beta=90^{\circ}$; cell $L=5 \mathrm{~mm}, s=4 \mathrm{~mm}$.

- no positive induced effect, as all the primaries are deposited in a single cell,

- minimized saturation effect (fig. 10).

Fig. 12 shows the mean pulse height per unit length measured at different track lengths -10 to $40 \mathrm{~mm}$ - and different amplifications. A linear dependance is observed up to $\approx 2 \mathrm{pC}$ in both cases [sect. 2.2, eq. (1)]:

- pulse height vs energy loss at fixed amplification,

- pulse height vs amplification at fixed energy loss.

The comparison of these data with those obtained from the $5.9 \mathrm{keV}$ line of an ${ }^{55} \mathrm{Fe}$ source provides the mean energy loss per unit length of this gas mixture

$\mathscr{E}_{0}=(3000 \pm 200) \mathrm{eV}$.

On the other hand, the same result was found from an ELS simulated by Monte Carlo meth$\mathrm{od}^{11}$ ): that is shown in fig. 13, which compares 
a measured to a simulated spectrum at equivalent conditions.

\section{Influence of the positive induced pulses}

Now let us come to this purely geometrical effect, which ought to occur each time a track crosses more than one wire cell.

The results are relative to the trajectories, $\beta=0^{\circ}, \alpha=15^{\circ}$, situation where all motions of negative and positive charges are synchroneous.

Measurements originate from ten adjacent wires, each of them providing a "one cell spectrum". Another spectrum, called $\Sigma$ spectrum, is obtained by doing, on each event, the sum of the ten pulse heights divided by ten.

\subsection{TyPiCAL RESUlts}

They correspond to the cell geometry

$L=12 \mathrm{~mm}, s=4 \mathrm{~mm}, \varnothing=20 \mu \mathrm{m}$,

- a one-cell spectrum has a truncated shape (fig. 14a),

- this truncation implies an inefficiency per wire, equal in this case to $\approx 20 \%$,
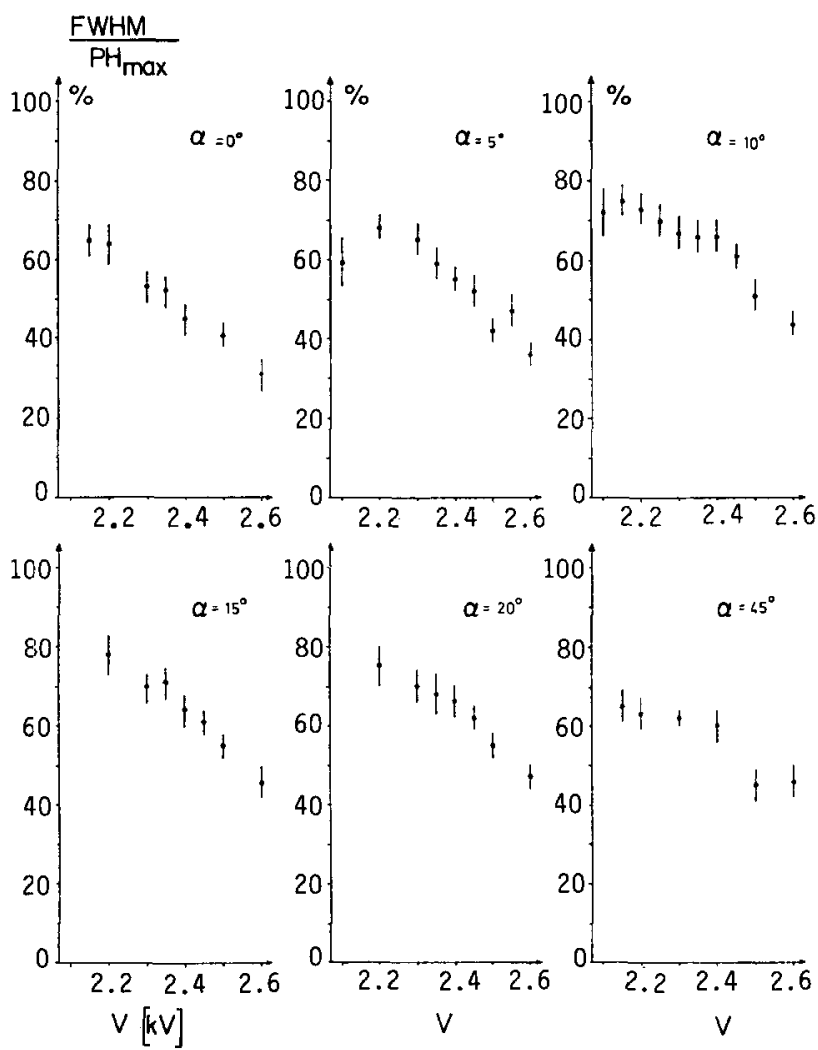

Fig. 11. Energy resolution vs high voltage at different angle $\alpha$ at $\beta=90^{\circ}$, cell : $L=5 \mathrm{~mm}, s=4 \mathrm{~mm}$.
- this inefficiency is found approximatively unchanged for amplification up to $3 \times 10^{5}$,

- the $\Sigma$ spectrum is not truncated (fig. 14b),

- the respective means are

$$
\begin{aligned}
\langle\mathrm{PH}(1)\rangle & =(840 \pm 80) \mathrm{eV}, \\
\langle\mathrm{PH}(\Sigma)\rangle & =(680 \pm 70) \mathrm{eV},
\end{aligned}
$$

whereas we expect for a track length of $4 \mathrm{~mm}$ (sect. 4.5 , proportional regime)

$\mathscr{E}=(1200 \pm 100) \mathrm{eV}$.

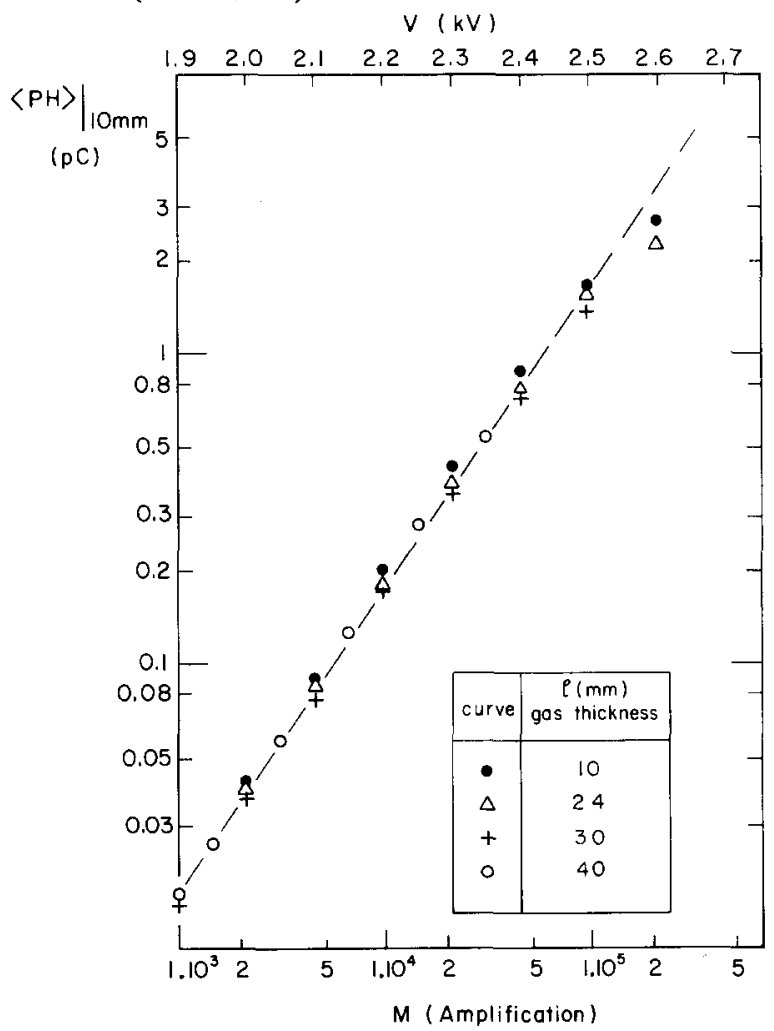

Fig. 12. Proportional regime. Normalized pulse height vs high voltage measured at different gap width; $\beta=90^{\circ}, \alpha=15^{\circ}$.

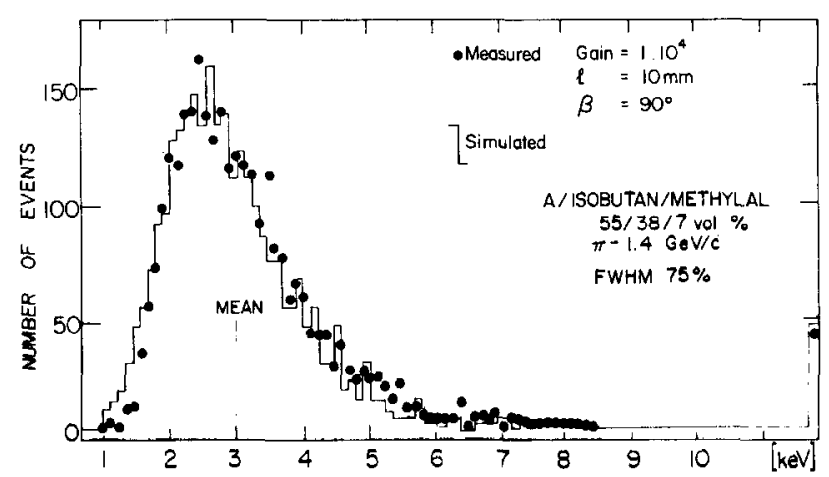

Fig. 13. Monte Carlo simulation of an ELS in $10 \mathrm{~mm}$ of gas mixture; the fluctuations in amplifications are taken into account. Comparison to a measured spectrum. 

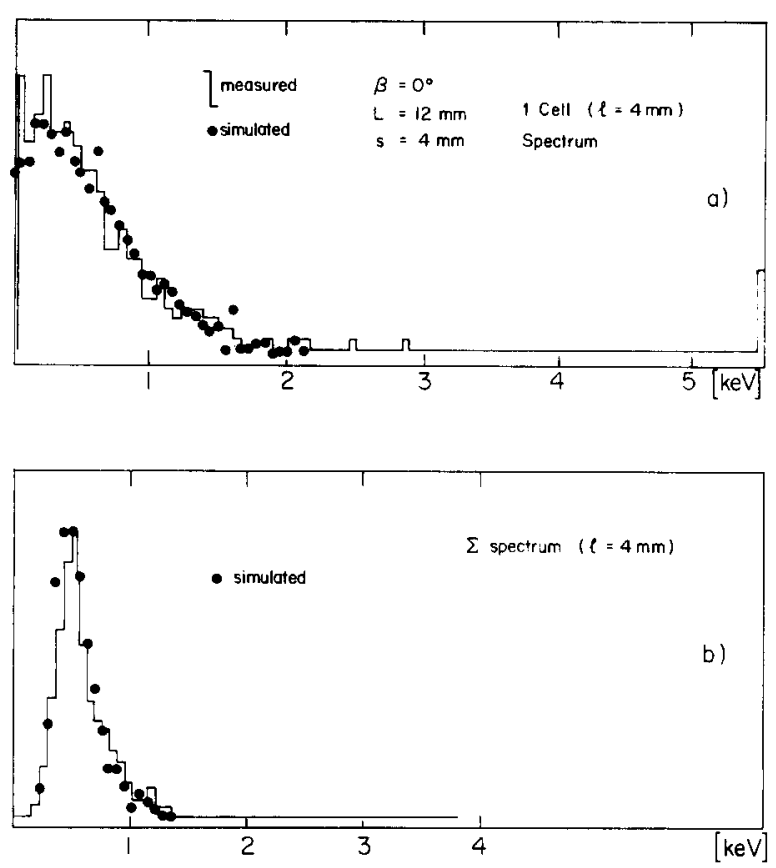

Fig. 14. Spectra showing the influence of positive induced pulses: $\quad \beta=0^{\circ}, \quad \alpha=15^{\circ} ; \quad$ cell : $L=12 \mathrm{~mm}, \quad s=4 \mathrm{~mm}$; gain $\approx 1 \times 10^{5}$. The $0 \mathrm{dc}$ level of the electronics has been displaced. (a) One cell spectrum, measurement and simulation with positive induced influence, (b) $\Sigma$ spectrum (10 cells).

In the calculation of the mean of the one cell spectrum, the loss in area due to the truncation has less influence than the loss in number of events due to the $20 \%$ inefficiency; this provides a too small divisor and explains why $\langle\mathrm{PH}(1)\rangle\rangle\langle\mathrm{PH}(\Sigma)\rangle$ since the $\Sigma$ spectrum is not truncated and the efficiency is $\approx 100 \%$.

The factors by which one has to correct the means of these PHS, in order to find again the mean of the corresponding ELS are

$f(1)=\frac{\mathscr{E}}{\langle\mathrm{PH}(1)\rangle}, \quad f(\Sigma)=\frac{\mathscr{E}}{\langle\mathrm{PH}(\Sigma)\rangle}$,

respectively equal to 1.43 and 1.77 . Fig. 15 shows that such a factor acts correctly at different gas amplifications.

\subsection{Simulation of Positive induCED pulses}

The influence of the positive induced pulses was simulated by a Monte Carlo program, according to the following steps:

a) A sample of $n$ energy losses $\left(a_{1}, a_{i}, a_{n}\right)$ is randomly drawn from the ELS, which corresponds to the track length $s$ : this simulates a track which crosses the cells 1 to $n$ at $\beta=0^{\circ}$.

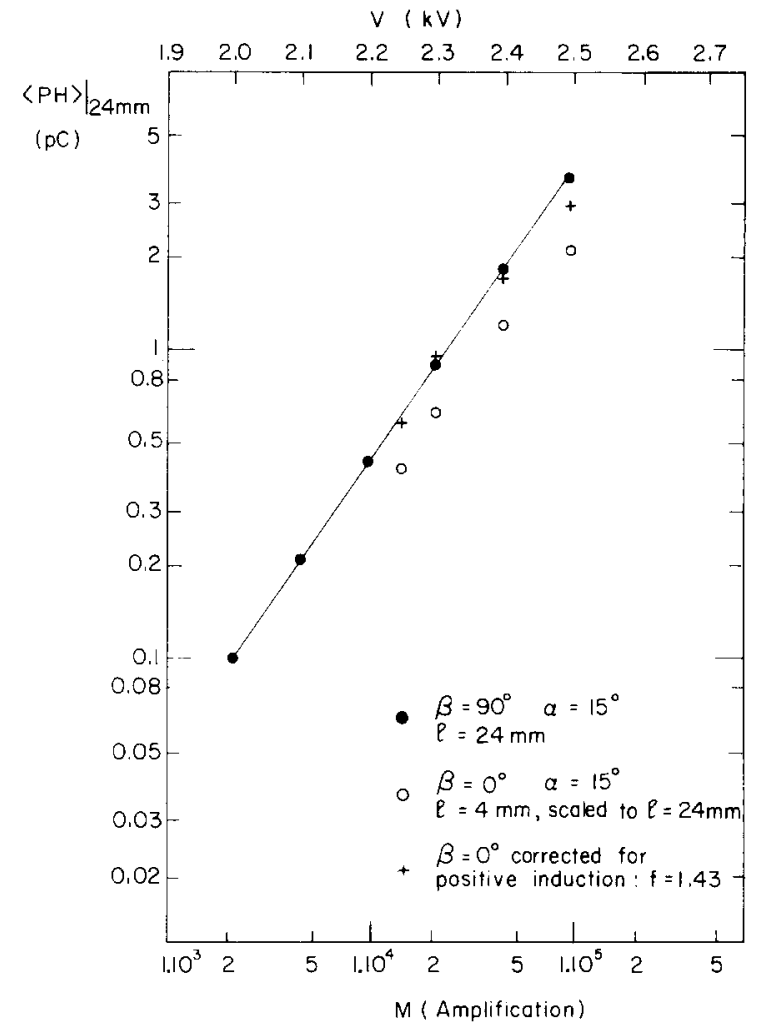

Fig. 15. Application of correction factor for positive induced pulses effect: curve $1: \beta=90^{\circ}, \alpha=15^{\circ}$; cell: $L=12 \mathrm{~mm}$, $s=4 \mathrm{~mm} ;\langle\mathrm{PH}\rangle \mathrm{s}$ correspond to $24 \mathrm{~mm}$ track length; curve 2 : $\beta=0^{\circ}, \alpha=15$; cell : $L=12 \mathrm{~mm}, s=4 \mathrm{~mm}$; measured: $\langle\mathrm{PH}\rangle \mathrm{s}$ correspond to $4 \mathrm{~mm}$ track length; plotted: normalized at $24 \mathrm{~mm}$, one cell spectrum. By multiplying measurements of curve 2 by the correction factor 1.43 , one finds again the proportional regime of curve 1 .

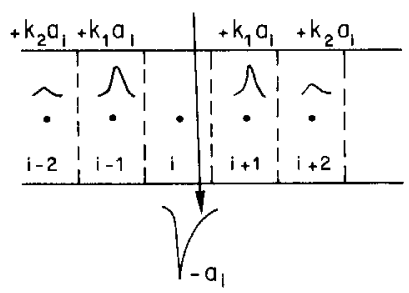

Fig. 16. Illustration of the positive induction coefficients.

b) Assuming that the pulse height at a given cell $\mathrm{i}$ is given by

$a_{i}-k_{1}\left(a_{i-1}+a_{i+1}\right)-k_{2}\left(a_{i-2}+a_{i+2}\right)-\ldots$,

the Monte Carlo program generates all the one cell spectra and the $\Sigma$ spectrum.

The coefficients of positive induction $\left(k_{1}, k_{2} \ldots\right)$ used in eq. (3), are defined as follows (fig. 16): a particle traversing only one cell $i$, at $\beta=90^{\circ}$ will induce a pulse height $\left(-a_{i}\right)$ on this particular wire 


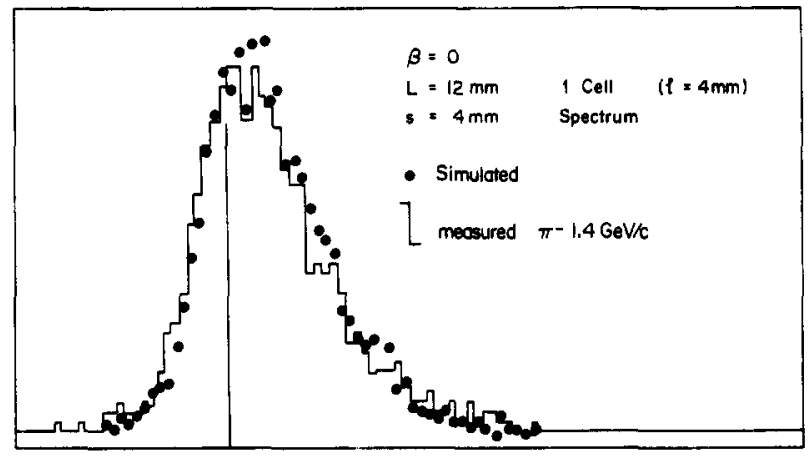

O DC LEVEL

Fig. 17. Spectrum with positive induced pulses influence: $\beta=0^{\circ}, \alpha=15^{\circ}$; cell : $L=12 \mathrm{~mm}, s=14 \mathrm{~mm}$. The dc level of the electronics has been shifted. The full spectrum can be seen and compared to the Monte Carlo simulation.

and respectively $+k_{1} a_{i},+k_{2} a_{i}$ on the first, second ... adjacent wires, symmetrically to the wire $i$.

These coefficients have been measured, following this definition, for different cell geometries at $\beta=90^{\circ}$ (table 3 ).

\section{Results}

- The measured shapes and inefficiency are correctly reproduced as shown in figs. $14 a, b$.

- The complete shape of such a PHS can be-observed experimentally by polarizing conveniently the dc input level of the electronics. Fig. 17 shows the comparison between measurement and simulation.

\subsection{Cell geometry influence}

We have repeated measurements and simulations for different cell geometries. Tables 3 and 4 summarize the results.

In order to minimize the induction effect, results of table 4 indicate that one has to keep the ratio $L / s$ as small as possible. A larger $s$ minimizes the inefficiency owing to a better energy resolution.

\section{Conclusions}

These results allow us to draw some conclusions about the influence of the track orientation on the energy loss measurements in MWPC:

1) In case of tracks traversing MWPC gaps $\left(\beta \approx 90^{\circ}\right)$, the determination of the angle $\alpha$ is always required with an accuracy of few degrees even in devices mainly devoted to $\mathrm{d} E / \mathrm{d} x$ measurements. In fact, it has been found that at amplification of the order of
TABLE 3

Coefficient of positive induction at different cell geometries. The electronic cross talk was: $+0.3 \%$ on the first adjacent of a negative signal

\begin{tabular}{lcccc}
\hline$L(\mathrm{~mm})$ & 5 & 12 & 20 & 15 \\
$s(\mathrm{~mm})$ & 4 & 4 & 4 & 8 \\
$k_{1}$ & 0.080 & 0.15 & 0.19 & 0.12 \\
$k_{2}$ & 0.025 & 0.06 & 0.07 & 0.03 \\
$k_{3}$ & & 0.02 & 0.03 & \\
\hline
\end{tabular}

TABLE 4

Correction factors for positive induced pulses effect and inefficiency per wire for different cell geometries.

\begin{tabular}{lccc}
\hline$L(\mathrm{~mm})$ & 5 & 12 & 15 \\
$s(\mathrm{~mm})$ & 4 & 4 & 8 \\
$f(1)$ simulated & 1.27 & 1.46 & 1.41 \\
measured & 1.30 & 1.43 & 1.49 \\
$\quad-$ & 1.31 & 1.74 & 1.43 \\
$f(\Sigma) \quad-$ & 1.35 & 1.77 & 1.47 \\
Inefficiency & 0.04 & $\sim 0.20$ & $\sim 0.02$ \\
\hline
\end{tabular}

$5 \times 10^{3}$ to $2 \times 10^{4}$, the saturation effect may reduce the pulse height by 20 to $60 \%$ when $\alpha$ varies from $20^{\circ}$ to $0^{\circ}$ - track orthogonal to the wire.

2) In case of tracks parallel to the wire plane $\left(\beta=0^{\circ}\right)$, the saturation effect due to $\alpha$ is much less important, although the saturated regime begins at a lower pulse height $(0.4 \mathrm{pC})$ than in the preceding case (1-2 pC).

3) A model which considers the time sequence of the avalanches together with the positive charge density was proposed to describe the observed phenomena: it will be developed quantitatively in the future.

4) When a track crosses many cells in one gap, the correction factor for induced positive pulses effect can also be important: it depends only on the cell geometry. For $L / s$ going from 1.25 to 3 , the factor increases from 1.3 to 1.7. An inefficiency per wire is also observed and can reach $20 \%$.

$5)$ In a jet chamber geometry, where the tracks always cross many adjacent cells $\left(\beta=0-10^{\circ}\right)$, one should only consider the relative variation of these factors due to the changes in the cell geometries along a sector.

We wish to thank A. Minten for his continued support, and P. G. Innocenti for his efficient help for this work. 
We are indebted to J. H. Cobb, H. G. Fischer, P. Hanke, P. G. Innocenti, F. Ranjard and O. Ullaland for many valuable discussions and advice.

We gratefully acknowledge J. C. Santiard for his essential contribution to the electronic design and G. Dinkel and C. Rivoiron for the excellent work in the chamber construction.

\section{References}

1) See for example the following papers, presented at this conference: J. Heintze, Drift chambers and recent developments; W. Farr, B. Granz, J. Heintze, R. D. Heuer, P. Lennert, T. Nozaki, H. Rieseberg and A. Wagner, A drift chamber system for the detection of narrow particle jets from $\mathrm{e}^{+} \mathrm{e}^{-}$reactions; C. Fabjan, H. B. Jensen, J. Lindsay, J. O. Petersen, F. Piuz, F. Ranjard, E. Rosso, A. Rudge, S. Serednyakov and W. Willis, A drift chamber vertex detector for intersecting storage rings.

2) See for example: Proposal for a PEP Facility based on the time projection chamber, John Hopkins-LBL-UCLA-UCRYale Collaboration; Proposal for a $4 \pi$ solid angle detector for the SPS used as a proton antiproton collider at a centre of mass of $540 \mathrm{GeV} /$ SPSC 78-6, SPSC/P92.

3) These references give a detailed review of all physical phenomena involved in MWPC, their properties and extensive bibliography: F. Sauli, Principles of operation of multiwire proportional and drift chambers, Lectures given at the academic training programme of CERN 1975-1976, Yellow Report CERN 77-9; P. Rice-Evans, Spark, streamer proportional and drift chambers (Richelieu, London 1974).

4) G. Schultz, CERN Internal Report EP 76-19, (1976); A. Breskin, G. Charpak, F. Sauli, M. Atkinson and G. Schültz, Nucl. Instr. and Meth. 124 (1975) 189.

5) H. G. Fischer, F. Piuz, O. Ullaland, Proc. Int. Meeting on Proportional and drift chambers, Dubna (1975).

6) J. C. Santiard, Charge amplifier in semi-hybridation, Internal Note CERN/EP/JCS/ef (7 October 1976); B. Righini and J. Schiari, CERN/EP Report no. 53 (January 1978).

7) M. Spiro, CERN-p̄ note 34 (1977).

8) S. C. Brown, Basic data of plasma physics (Wiley, New York 1959); L. G. Christophorou, Atomic and molecular radiation physics (Wiley, New York 1971).

9) M. Panter, Diplomarbeit (Institut für Experimentelle Kernphysik, Universität Karlsruhe, 1977).

10) H. Frehse, M. Heiden, M. Panter and F. Piuz, these proceedings.

11) J. H. Cobb et al., Nucl. Instr. and Meth. 133 (1976) 315. 